\title{
VALIDATION AND DETECTION OF SEX-SPECIFIC MARKERS IN JOJOBA (SIMMONDSIA CHINENSIS) PLANTS IN SAUDI ARABIA
}

\author{
BAFEEL, S. ${ }^{*}-$ BAHIELDIN, A. \\ Department of Biological Sciences, Faculty of Science, King Abdulaziz University, Jeddah, \\ Kingdom of Saudi Arabia \\ *Corresponding author \\ e-mail: sbafil@kau.edu.sa \\ (Received $10^{\text {th }}$ Feb 2020; accepted $6^{\text {th }}$ May 2020)
}

\begin{abstract}
The main target of the present study is the validation of sex-specific markers previously described and the detection of new markers for further use in breeding programs of jojoba in Saudi Arabia. The used molecular approaches included random amplified polymorphic DNA (RAPD), intersimple sequence repeat (ISSR), Cleavage-amplified polymorphic sequence (CAPS) and amplified fragment length polymorphism (AFLP). RAPD and ISSR analyses were not useful in determining the sex of jojoba. CAPS with the J888 marker indicated the presence of two new sex-specific SNPs and consequent presence of several sex-specific markers. AFLP analysis successfully resulted in the recovery of several male- and female-specific markers. Whole genome sequencing was also done to detect new and validate already-known sex-specific markers. Use of NUCmer module resulted in no new sex-specific markers. BLAST analysis to validate sequence-related amplified polymorphism (SRAP) marker indicated its existence. Analysis of the possible functioning of $O G I / M e G I$ sex determination system in jojoba was not proven. In conclusion, a number of sex-specific markers were detected in jojoba plants growing in Saudi Arabia might help promoting the biofuel production industry in Saudi Arabia and the Arab region as a new approach to be adopted in the near future.
\end{abstract}

Keywords: RAPD, CAPS, AFLP, WGS, BLAST, NUCmer, SRAP, OGI/MeGI

\section{Introduction}

Biofuels can be either bioalcohol or biodiesel. The latter is made of renewable resources that are generated from plants like jojoba and jatropha (Pinzi et al., 2009). Comparing biofuels to fossil fuels indicates that the first reduces pollution and greenhouse effects. Jojoba (Simmondsia chinensis (Link) C. K.) is a perennial and plant native to Arizona, southern California and northern Mexico (Benzioni, 1995). The plant is dioecious, e.g., bearing male and female flowers on different plants with the axillary inflorescences of male plants harbor a number of 3-20 flowers, while axillary inflorescences of female plants harbor single flower (Ince and Karaca, 2011). The use of jojoba in biofuel production is advantageous because the plant is non-edible, unlike corn or sugarcane, and can be irrigated with low quality water and cultivated in areas with high levels of drought, salinity and heat. Jojoba possesses unique favorable properties, such as low acidity and viscosity, good oxidation stability and its oil possesses $<3 \%$ triglyceride and is highly resistant to oxidation (El-Mallah and El-Shami, 2009). Jojoba oil has several applications that make it economically feasible (Passerini and Lombardo, 2000). It is used in lubricants, pharmaceuticals and cosmetics, besides its potential use in industry, e.g., plastics, printer ink, surfactants and leather lubricants (Inca and Karaca, 2010).

The most common method for jojoba breeding is the non-sexual propagation along with the selection of plants with desirable characteristics (Vaknin et al., 2003; Tobares et al., 2004; Benzioni et al., 2005). However, there is a shortage of yield with the use of 
clones or cuttings in commercial plantations, therefore, the application of supplemental pollination results in an increased seed yield (Coates et al., 2006). Further analysis indicated that selection of appropriate male and female genotypes for plantation and breeding studies is mandatory. However, further recovery of low yield results mainly from the high female to male plant ratio (Benzioni and Ventura, 1998; Coates et al., 2006). The plantation with seeds usually results in $84 \%$ male and $16 \%$ female plants whereas only 10\% male plants are desirable for optimal yield (Agarwal et al., 2008). This ratio can be easily manipulated by planting male plants distantly far from the existing females. For economical plantation of jojoba, there is a need to identify the sex of the plant at the seedling stage. Early identification of sex will also lead to increased efficiency of a plantation program by allowing proper layout of field arrangement of male and female seedlings and early elimination of unwanted plants.

Unfortunately, sex can be determined morphologically, as indicated earlier, at mature stages when plants are 3-year-old or older, while sex is difficult to be determined at earlier stages. However, sex in young seedlings was determined at the molecular level so far but with less success. Therefore, it is important to find effective molecular or DNA markers that can reliably be used in the easy determination of sex at early stages of plant development. DNA markers have several advantages as they exhibit high allelic variation, being neutral to the environmental conditions (Tan et al., 2003; Karaca et al., 2004), besides being used in sex identification in several plant crop species including basket willow (Salix viminalis L.) (Alstrom-Rapaport et al., 1998), Atriplex garrettii (Ruas et al., 1998), Viola pubescens Aiton (Culley and Wolfe, 2000), nutmeg (Myristica fragrans Houtt.) (Shibu et al., 2000), Pistacia sp. (Kafkas et al., 2001), Mercurialis annua (Khadka et al., 2002), hemp (Torjek et al., 2002), Eucommia ulmoides Oliv (Xu et al., 2004), hop (Humulus lupulus L.) (Danilova and Karlov, 2006), Encephalartos natalensis (Prakash and van Staden, 2006), Carica papaya (Chaves-Bedoya and Nunenz, 2007), Carica papaya (Gangopadhyay et al., 2007), bermudagrasses (Cynodon spp.) (Karaca and Ince, 2008), subtropical carrot (Jhang et al., 2010), Calamus simplicifolius (Li et al., 2010) and jojoba (Mohasseb et al., 2009; Ince et al., 2010; Agarwal et al., 2011; Ince and Karaca, 2011; Heikrujam et al., 2014; Kumar et al., 2019).

The present study aims at validating a number of sex-specific markers previously used in jojoba towards further usage in breeding programs of jojoba growing in Saudi Arabia. Results of this study might help determining sex in jojoba more efficiently, thus, promoting the biofuel production industry in Saudi Arabia and the Arab region, a new approach possibly adopted in the near future.

\section{Materials and methods}

\section{DNA extraction}

The analysis involved a number of five male and five female plants growing at King Abdulaziz Farm station at Hada El-Sham near Jeddah, Kingdom of Saudi Arabia. Flashfrozen leaf materials from individual plants were crushed into a fine powder in a microcentrifuge tube using a sterilized metal rod and DNAs were isolated from leaves following the modified procedure of Gawel and Jarret (1991). Isolated DNAs were, then, treated with RNase A $(10 \mathrm{mg} / \mathrm{ml})$ and incubated at $37{ }^{\circ} \mathrm{C}$ for $30 \mathrm{~min}$ to remove RNA contaminants. DNA concentrations were estimated by measuring optical density at $260 \mathrm{~nm}$ according to the equation: DNA concentration $(\mathrm{ug} / \mathrm{ml})=0 D 260 \mathrm{X} 50 \mathrm{x}$ dilution factor. 


\section{Random amplified polymorphic DNA (RAPD) and inter-simple sequence repeat (ISSR) analyses}

For RAPD analysis, a number of 20 random 10mer primers (Operon Technology, USA) from groups $\mathrm{A}, \mathrm{B}$ and $\mathrm{C}$ was used in determining sex in male and female jojoba plants (Appendix 1). PCR was carried out in $25 \mu 1$ reaction volume containing 1x PCR buffer, $4 \mathrm{mM} \mathrm{MgCl} 2,0.2 \mathrm{mM}$ dNTPs, 20 pmole primer, 2 units Taq DNA polymerase and $25 \mathrm{ng}$ template DNA. PCR amplification was performed in a Perkin Elmer 2400 thermocycler (Germany), programmed to fulfill 40 cycles after an initial denaturation cycle for $4 \mathrm{~min}$ at $94{ }^{\circ} \mathrm{C}$. Each cycle consisted of a denaturation step at $94{ }^{\circ} \mathrm{C}$ for $1 \mathrm{~min}$, an annealing step at $37^{\circ} \mathrm{C}$ for $2 \mathrm{~min}$, and an extension step at $72^{\circ} \mathrm{C}$ for $2 \mathrm{~min}$, followed by a final extension cycle for $7 \mathrm{~min}$ at $72^{\circ} \mathrm{C}$.

For ISSR, 14 primers were used in detecting polymorphic bands between male and female plants (Appendix 1). PCR analysis was performed in $25 \mu \mathrm{l}$ reaction and amplification was programmed to fulfill 40 cycles after an initial denaturation cycle for 4 min at $94{ }^{\circ} \mathrm{C}$. Each cycle consisted of a denaturation step at $94{ }^{\circ} \mathrm{C}$ for $1 \mathrm{~min}$, an annealing step at $40{ }^{\circ} \mathrm{C}$ for $80 \mathrm{~s}$, and an extension step at $72{ }^{\circ} \mathrm{C}$ for $2 \mathrm{~min}$, followed by a final extension cycle for $7 \mathrm{~min}$ at $72^{\circ} \mathrm{C}$. Amplicons were run on agarose gel (Wide Mini-Sub Cell GT Systems, Bio-Rad) at 120 V/50 mA (PowerPac Universal Power Supply, Bio-Rad) for $45 \mathrm{~min}$, then gel stained with ethidium bromide $(0.3 \mathrm{ug} / \mathrm{ml})$ and visually examined with an UV transilluminator and photographed using a CCD camera (UVP, UK).

\section{Cleavage-amplified polymorphic sequence (CAPS)}

CAPS was generated for the J888 marker using the primer pair 5 AGACCCAGAGCACACACAGC-3' (forward) and 5AGACCCAGAGGATGAGGAATG-3` (reverse) to recover 888 bp as previously described (Ince and Karaca, 2011). The amplicons were shipped to BGI, China for Sanger sequencing and sequences were analyzed via using SnapGene ${ }^{\circledR}$ in order to detect Single Nucleotide Polymorphism (SNP) markers and make predicted restriction analysis towards the detection of possible polymorphic sex-specific bands.

\section{Amplified fragment length polymorphism (AFLP)}

AFLP analysis was performed using the two combinations EcoRI-GC/MseI-GCG and EcoRI-TAC/MseI-GCG of the AFLP Analysis System I (Invitrogen, cat. no. 10544013) following manufacturer's protocol. Genomic DNAs of the five male and five female samples were digested with EcoRI and MseI restriction enzymes in which EcoRI and MseI adapters were ligated to the digested DNA fragments. Pre-amplification was carried out using EcoRI primer plus one extension base at the 3' position ( $\mathrm{G}$ or $\mathrm{T}$ ) and MseI primer plus one extension base at the 3' position $(G)$ to amplify fragments that contain complementary sequences.

\section{Whole genome sequencing}

Whole genome sequencing was done for one male and one female randomly-selected jojoba samples at BGI, China. About 60 million 100-bp paired-end reads were generated from sequencing libraries with 500-bp insert. Data were filtered to remove the low-quality reads and trim adaptor using Fastp package 
(https://www.ncbi.nlm.nih.gov/pmc/articles/PMC6129281/). The remaining sequencing reads from the two samples were de novo assembled via velvet (v1.2.10) without length cutoff as described (https://www.ebi.ac.uk/ Zerbino/velvet/). Contigs recovered from the two sexes were further used in detecting either new or previously-known sexspecific markers in jojoba. The raw data was deposited in the Short Read Archive database (BioProject ID PRJNA603451) of the NCBI.

\section{Detection of new sex-specific markers}

Multiple contig alignment of the genomes of the two sexes was done using NUCmer module 3.0 (NUCleotide MUMmer, part of mummer software) to determine the maximal unique matches of a given length (350-500 bp) between the two input sequences, a step to increase the overall coverage of the alignment (Kurtz et al., 2004). A number of 100 contigs of each genome showed high matching in DNA sequences. Contig pairs with high similarity ( $90 \%)$ and unique sex-specific areas of 350-500 bp (sequence similarity/difference criteria) were selected and sex-specific primers were generated (Appendix 1). PCR was performed using ready master mix (BioTaq Green Master Mix, Promega) and conditions were $95{ }^{\circ} \mathrm{C} / 5 \mathrm{~min}$ (initial denaturation), $95^{\circ} \mathrm{C} / 30 \mathrm{~s}, 52{ }^{\circ} \mathrm{C} / 45 \mathrm{~s}$ and $72{ }^{\circ} \mathrm{C} / 1 \mathrm{~min}$ (40 cycles), $72{ }^{\circ} \mathrm{C}$ (final extension), then reaction was held at $4{ }^{\circ} \mathrm{C}$. Amplicons were run on agarose gel, stained with ethidium bromide $(0.3 \mathrm{ug} / \mathrm{ml})$, then visually examined and photographed.

\section{Detection of previously-known sex-specific markers}

Resulted contigs from both sexes were blasted (ncbi-BLAST v 2.10.0) against previously-known sex-specific sequences referring to an ISSR marker namely UBC8071200 (Sharma et al., 2008; Heikrujam et al., 2014), sequence-related amplified polymorphism (SRAP) (Kumar et al., 2019) determining sex in jojoba as well as OGI/MeGI system determining sex in persimmons (Diospyros spp.) (https://www.ncbi.nlm.nih.gov/nuccore/KM408640) (Akagi et al., 2014).

\section{Results and discussion}

Production of jojoba has spread in many regions of the world (Benzioni et al., 2005) including the Middle East. However, no previous comparative analysis has been made at the molecular genetic level to detect genetic distances among genotypes existing in different regions. Therefore, there is no guarantee that the genetic makeup of the genotypes in Saudi Arabia will harbor the same molecular markers published elsewhere.

In the present study, several types of molecular markers using specific primers were proven not to be useful in determining sex in jojoba plants growing in Saudi Arabia (Appendix 2). As models, amplicons of two RAPD (with A02 and B09 primers) and two ISSR (with 544A and HB11 primers) are shown in Appendix 2. We speculate one reason for the failure to detect the known sex-specific RAPD or Td-PCR markers is that these two types of markers are likely genotype-specific. Identification of molecular marker for ascertaining sex of plants at seedling stage is vital for optimal plantation of dioecious plants including jojoba. This approach has previously been followed in many plants including Pistacia vera (Hormaza et al., 1994), Carica papaya (Deputy et al., 2002) and Mercurialis annua (Khadka et al., 2002) using random amplified polymorphic DNA (RAPD) approach, while sex in plants like Asparagus officinalis 
(Reamson-Büttner et al., 1998), Cannabis sativa (Flachowsky et al., 2001; Peil et al., 2003) and Ficus fulva (Parrish et al., 2004) was determined using amplified fragment length polymorphism (AFLP) approach. Previous studies to detect sex-specific molecular markers in jojoba included a number of RAPD (Agrawal et al., 2007), touchdown polymerase chain reaction (Td-PCR) (Ince et al., 2010) and cleavageamplified polymorphic sequence (CAPS) (Ince and Karaca, 2011) markers. Out of 72 RAPD primers, only one primer, e.g., OPG-5 (5'-CTGAGACGGA-3`), produced a male-specific marker of $\sim 1.4 \mathrm{~kb}$ (Agrawal et al., 2007). Using Td-PCR, another malespecific marker, namely JMS900, was detected using a 10-mer primer (5AGACCCAGAG-3') (Ince et al., 2010). Mohasseb et al. (2009) indicated that one reason for the failure to generate reliable information from these two types of markers is that they might be vulnerable to PCR conditions and quality of the genomic DNA.

The previous results of CAPS with the J888 marker indicated that the $888 \mathrm{bp}$ digested with ClaI, HindIII and HinfI produced polymorphic fragments in male and female samples (Ince and Karaca, 2011). In the present study, this was not the case as digestion of the $888 \mathrm{bp}$ amplicon with these three restriction enzymes generated no polymorphic sex-specific bands (data provided upon request). Therefore, the J888 marker was sequenced for five male and five female samples and multiple sequence alignments of the forward and reverse sequences were done using Clostal Omega (Appendices 3 and 4, respectively). The results indicated the presence of two sexspecific SNPs shown in both the forward [sites $138(\mathrm{G}>\mathrm{A})$ and $330(\mathrm{C}>\mathrm{T})$ ] (Table 1 and Appendix 3) and reverse [sites $609(\mathrm{C}>\mathrm{T})$ and $416(\mathrm{G}>\mathrm{A})$ ] (Table 1 and Appendix 4) directions of the clean sequences.

Table 1. List of sex-specific SNPs either lying within restriction sites (colored boxes) or not (clear boxes) across five male (J1-J5) and five female (J6-J10) samples existing in the CAP marker J888. Sanger sequencing was done for J888 amplicon at the two (forward and reverse) directions. SNPs are mainly $C / T$ and $A / G$, while restriction sites are for Bsp119I

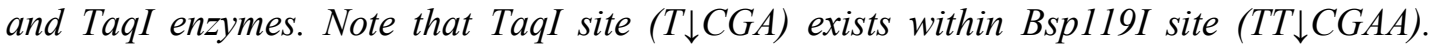
Red $=$ male, blue $=$ female. Multiple sequence alignments of the forward and reverse sequences of $J 888$ marker using Clostal Omega are shown in Appendices 1 and 2, respectively

\begin{tabular}{|c|c|c|c|c|c|c|c|c|c|c|c|}
\hline Primer & $\mathbf{J 1}$ & $\mathbf{J 2}$ & $\mathbf{J 3}$ & $\mathbf{J 4}$ & $\mathbf{J 5}$ & J6 & $\mathbf{J} 7$ & $\mathrm{J8}$ & J9 & $\mathbf{J 1 0}$ & SNP position (type)/restriction site \\
\hline \multirow{4}{*}{ Forward } & A & A & A & A & A & A & A & A & A & $\mathrm{T}$ & $44(\mathrm{~A}>\mathrm{T})$ \\
\hline & G & $\mathrm{G}$ & G & $\mathrm{G}$ & G & A & A & A & A & A & $138(\mathrm{G}>\mathrm{A}) / \mathrm{TaqI}$ \\
\hline & $\mathrm{G}$ & $\mathrm{G}$ & G & $\mathrm{G}$ & $\mathrm{G}$ & G & $\mathrm{G}$ & G & $\mathrm{G}$ & A & $268(\mathrm{G}>\mathrm{A})$ \\
\hline & $\mathrm{C}$ & $\mathrm{C}$ & $\mathrm{C}$ & $\mathrm{C}$ & $\mathrm{C}$ & $\mathrm{T}$ & $\mathrm{T}$ & $\mathrm{T}$ & $\mathrm{T}$ & $\mathrm{T}$ & $330(\mathrm{C}>\mathrm{T}) / \mathrm{Bsp} 119 \mathrm{I} / \mathrm{TaqI}$ \\
\hline \multirow{5}{*}{ Reverse } & G & $\mathrm{G}$ & G & $\mathrm{G}$ & G & A & A & A & A & A & $416(\mathrm{G}>\mathrm{A}) / \mathrm{Bsp} 119 \mathrm{I} / \mathrm{TaqI}$ \\
\hline & $\mathrm{C}$ & $\mathrm{C}$ & $\mathrm{C}$ & $\mathrm{C}$ & $\mathrm{C}$ & $\mathrm{C}$ & $\mathrm{C}$ & $\mathrm{C}$ & $\mathrm{C}$ & $\mathrm{T}$ & $479(\mathrm{C}>\mathrm{T})$ \\
\hline & $\mathrm{C}$ & $\mathrm{C}$ & $\mathrm{C}$ & $\mathrm{C}$ & $\mathrm{C}$ & $\mathrm{T}$ & $\mathrm{T}$ & $\mathrm{T}$ & $\mathrm{T}$ & $\mathrm{T}$ & $609(\mathrm{C}>\mathrm{T}) / \mathrm{TaqI}$ \\
\hline & $\mathrm{T}$ & $\mathrm{G}$ & G & $\mathrm{G}$ & G & G & $\mathrm{G}$ & G & G & G & $678(\mathrm{G}>\mathrm{T})$ \\
\hline & A & $\mathrm{G}$ & G & $\mathrm{G}$ & G & G & $\mathrm{G}$ & G & G & G & $695(\mathrm{G}>\mathrm{A})$ \\
\hline
\end{tabular}

In addition, restriction analysis and maps of one randomly selected male (Appendix 5) and one selected female (Appendix 6) samples were detected using SnapGene ${ }^{\circledR}$. The latter software predicted the occurrence of several polymorphic sexspecific bands when the amplicon is digested with either restriction enzymes TaqI or 
Bsp119I (Fig. 1 and Appendix 7). These polymorphic bands are consequences of the two SNPs sites located within the male and female of the J888 marker sequences. For TaqI, two male-specific markers with 125 and $70 \mathrm{bp}$, while two female-specific markers with 173 and $90 \mathrm{bp}$ were detected. For Bsp119I, three male-specific markers with 80, 70 and $53 \mathrm{bp}$, while two female-specific markers with 123 and $90 \mathrm{bp}$ were detected. Note

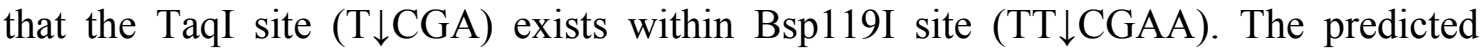
number of restriction fragments of TaqI enzyme is much higher than that of Bsp119I as the recognition site of the first is 4-base, while 6- base for the second. We expected that DNA sequences of the $90 \mathrm{bp}$ female-specific marker across the two restriction enzymes are the same. Then, we claim that we successfully detected two SNPs markers that resulted in the production of five male-specific and four female-specific markers when the J888 amplicon was digested with both TaqI and Bsp119I restriction enzymes.

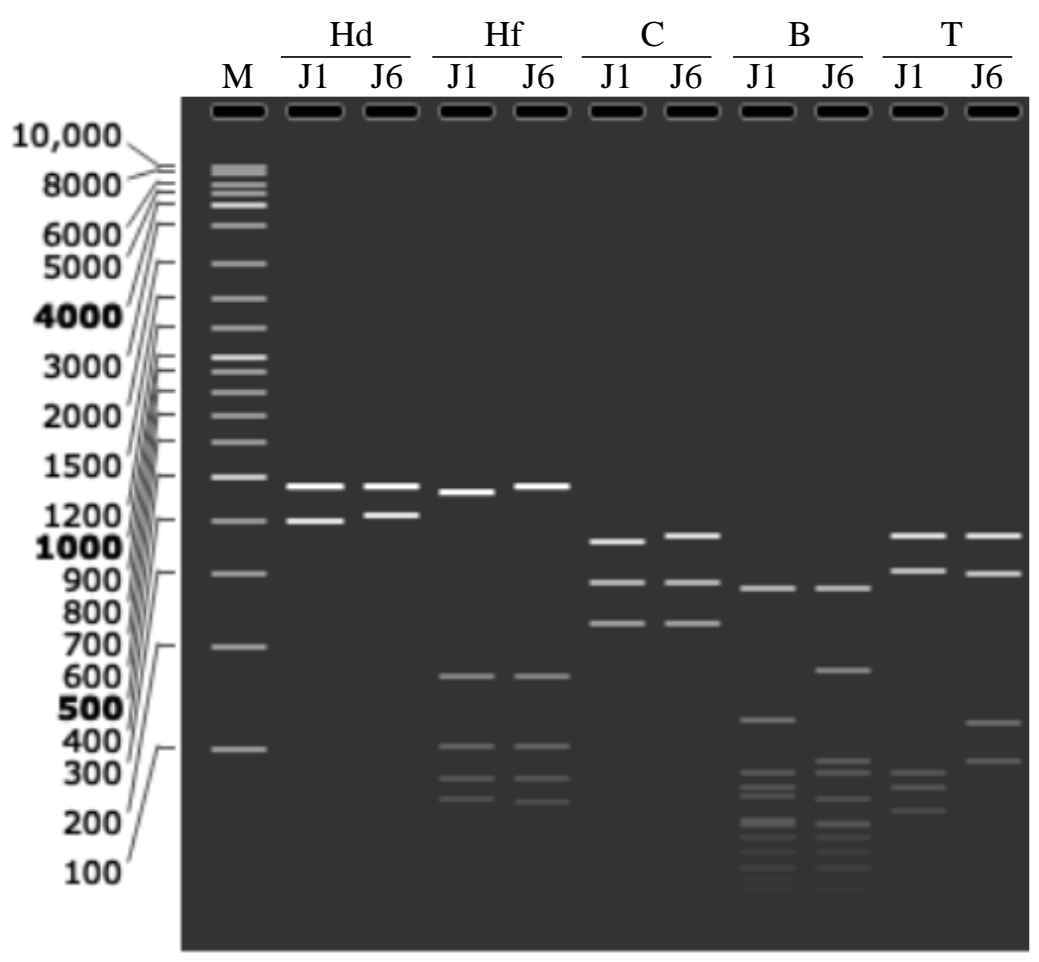

Figure 1. Predicted restriction analysis displayed on a virtual gel for one male (sample J1) and one female (sample J6) samples existing in the CAP marker J888. Restriction enzymes involved

HindIII (Hd), HinfI (Hf), ClaI (C), Bspl19I (B) and TaqI (T). Note that TaqI site (T\CGA) exists within Bsp119I site (TT\CGAA). $M=100 \mathrm{bp}$-ladder. Sizes of restriction fragments are shown in Appendix 3. Complete restriction maps of $J 888$ markers for the male and female samples are shown in Appendices 4 and 5. Restriction fragment sizes and maps were detected using SnapGene ${ }^{\circledR}$

AFLP is a reliable approach in detecting molecular markers in dioecious plants (Mwase et al., 2007), while RAPD, a dominant marker, has several drawbacks and lacks reproducibility (Mohasseb et al., 2009). Then, AFLP has a large chance to detect sexspecific markers in jojoba (Agarwal et al., 2011) as this type of markers allows the testing of large genomic fragments at once compared with the earlier types of molecular markers that test very small regions of the genome. Previous efforts utilizing AFLP in detecting sex-specific markers resulted in the recovery of two male-specific markers of 
$\sim 525$ and 325 bp using primer combinations EcoRI-GC/MseI-GCG and EcoRITAC/MseI-GCG, respectively, while only one female-specific marker of $\sim 270$ bp using the primer combination EcoRI-TAC/MseI-GCG. In the present study, the primer combination EcoRI-GC/MseI-GCG resulted in the recovery of five (428, 307, 190, 163 and $60 \mathrm{bp}$ ) male-specific and three (768, 401 and $25 \mathrm{bp})$ female-specific markers (Appendix 8), while the primer combination EcoRI-TAC/MseI-GCG resulted in the recovery of three (340, 196 and $56 \mathrm{bp})$ male-specific and three (425, 241 and $179 \mathrm{bp})$ female-specific markers (Appendix 9). We disconsidered sex-specific markers with $>25 \mathrm{bp}$. Dendrograms utilizing the two combinations resulted in the complete separation of the two sexes (Figs. 2 and 3). Then, we claim that this type of marker successfully determines sex in jojoba with high efficiency.
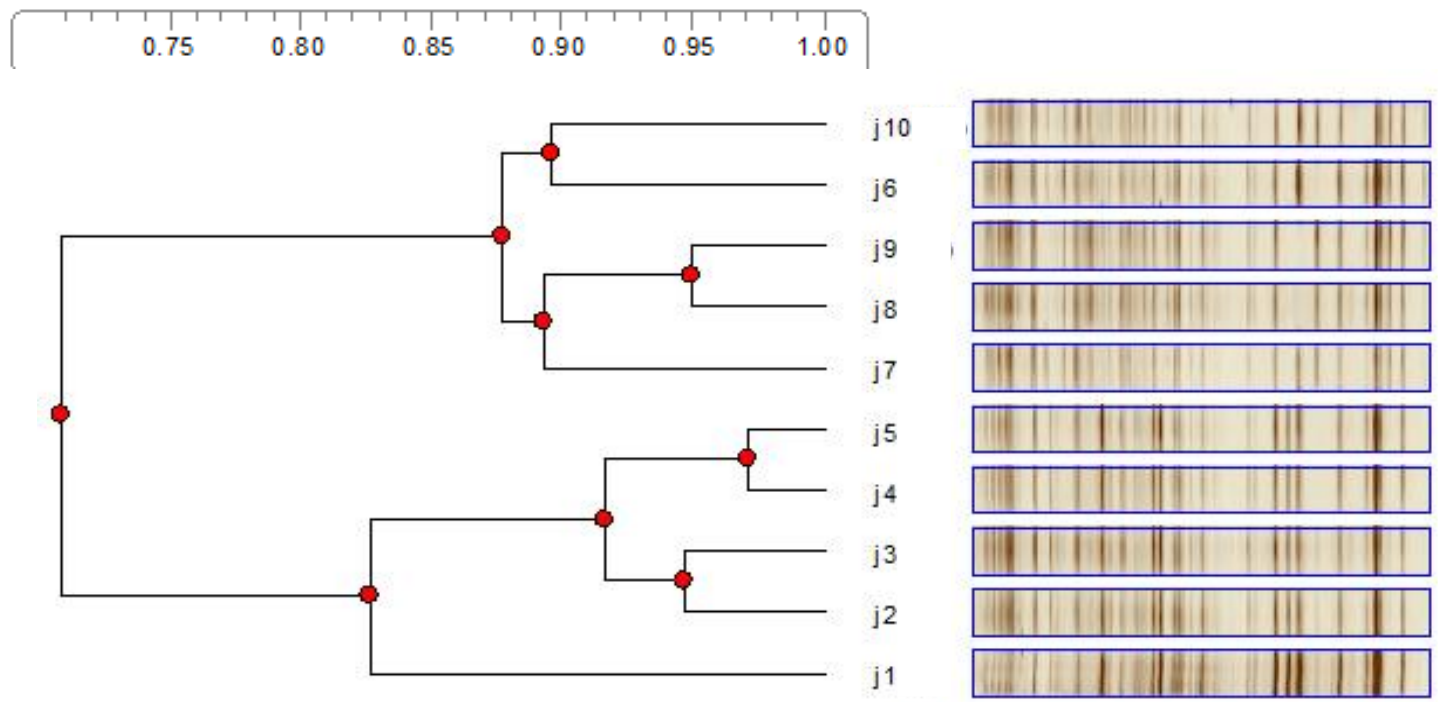

Figure 2. Dendrogram resulted from AFLP with the primer combination EcoRI-GC/MseI-GCG to describe the relationship between male (J1-J5) and female (J6-J10) banding patterns
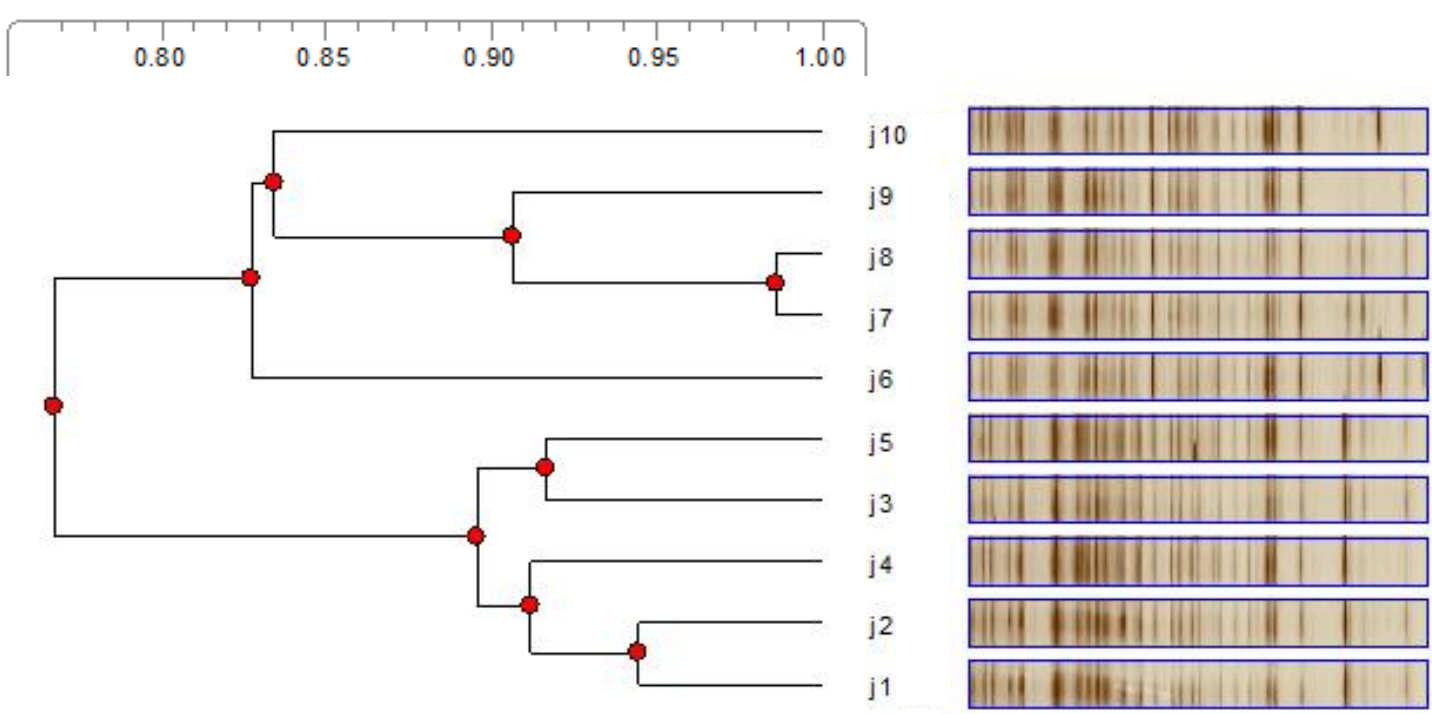

Figure 3. Dendrogram resulted from AFLP with the primer combination EcoRI-TAC/MseIGCG to describe the relationship between male (J1-J5) and female (J6-J10) banding patterns 
Multiple contig alignment of the genomes of the two sexes done using NUCmer module 3.0 (NUCleotide MUMmer, part of mummer software) indicated a number of 18 male and 17 female unique sequences of 350-500 bp. PCR with the designed sexspecific primer combinations was done for one male and one female samples and results indicated the occurrence of four male-generated ( $\mathrm{Rx} 9, \mathrm{Rx} 11, \mathrm{R} \times 26$ and $\mathrm{R} \times 33)$ and two female-generated ( $\mathrm{Rx} 1$ and $\mathrm{Rx} 4$ ) amplicons (Appendix 2). Interestingly, the malegenerated amplicons of Rx9 and Rx11 were supposed to be possible female-specific markers. PCR for five male and female samples indicated arbitrary inconsistent presence/absence of the four male and two female-generated amplicons in the two sexes (Appendix 2). We speculate that the failure to detect sex-specific markers via multiple contig alignment of the genomes of the two sexes and the use of MUMmer software is due to the need for larger yield or coverage of raw reads of genomes of the two sexes or due to the lack of appropriate binding sites in the template. Low coverage results in the presence/absence of many contig sequences that are not sex-specific. Therefore, we recommend repeating the whole genome sequencing of genomes of the two sexes, but requesting larger read coverage.

Two male-specific ISSR (Sharma et al., 2008; Heikrujam et al., 2014) and sequencerelated amplified polymorphism (SRAP) (Kumar et al., 2019) markers have also been validated in the present study. ISSR was previously reported to result in the recovery of a male-specific marker namely UBC-807 1200 utilizing primer UBC-807, while SRAP was reported to result in the recovery of a male-specific marker with an amplicon size of 396 bp utilizing primer combination Em14/Me10. In the present study, we have sequenced the whole jojoba male and female genomes and blasted the de novo assembled contigs of the two genomes against the published sequence of the malespecific ISSR marker in the NCBI (acc. no. HQ166029). The results indicated the presence of several fragments of the marker sequence in both male (Fig. 4 and Appendix 10) and female (Fig. 4 and Appendix 11) genomes. Results of the blasted male and female de novo assembled genomes against the published sequence of the SRAP male-specific marker of Kumar et al. (2019) with primer combination Em14/Me10 are shown in Appendices 12 and 13, respectively. The results proved that the DNA sequence of the SRAP marker was almost completely detected in the male sample (Fig. 5), while completely absent in the female sample. We concluded that the SRAP marker can successfully determine males in jojoba with high efficiency, while the UBC$807_{1200}$ marker was not proven to be male-specific.

Dioecy is often associated with the occurrence of sex chromosomes in plant and the presence of genetic determinants of sex (Ming et al., 2011; Renner, 2014; Charlesworth, 2016). After the era of genomics and whole genome sequencing (WGS), it is now possible to decipher the architecture of sex chromosomes in several dioecious plants (Liu et al., 2004; Ming et al., 2011; Wang et al., 2012, 2013; Charlesworth, 2016; Kazama et al., 2016; Harkess et al., 2017; Muyle et al., 2017). Genetic determinants of sex were deciphered in some species, including persimmons (Diospyros spp.) (Akagi et al., 2014), garden asparagus (Asparagus officinalis L.) (Harkess et al., 2017) and kiwifruit (Actinidia spp.) (Akagi et al., 2018). Sex was determined in persimmons through the action of one single non-coding RNA gene located on the Y chromosome namely $O G I$ gene (Yang et al., 2019). This gene produces a small-RNA sequence that targets an autosomal counterpart gene namely MeGI (Yang et al., 2019). The latter is thought to be a single integrator of sex expression (Akagi et al., 2014, 2016; Henry et al., 2018; Yang et al., 2019). As OGI gene is located on Y chromosome, we expected 
that if this sex determination system exists in jojoba, then existence of $O G I$ gene will be a male-specific marker, while we expect that the $M e G I$ gene exists in the two sexes. BLAST results of the de novo assembled contigs in the present study against the published sequences of the $O G I$ and MeGI genes are shown for male (Appendix 14 and 15, respectively) and female (Appendix 16 and 17, respectively) jojoba genomes. The sequences of $O G I$ and $M e G I$ genes of persimmon are available in the DDBJ database, with the Illumina reads for the mRNA-Seq analysis deposited in the Short Read Archive database (BioProject ID PRJDB7688) of the NCBI. The BLAST results indicated that $O G I$ gene was not detected in either jojoba sexes, while $M e G I$ gene was detected in 12 and six contigs of the jojoba male and female samples, respectively. Prior information neither confirmed the existence of Y chromosome in male plants of jojoba, nor proved the existence of $O G I / M e G I$ system in jojoba. Existence of $M e G I$, while lack of $O G I$ is a question remains to be answered. In general, we confirmed that the published $O G I / M e G I$ system does not exist to drive sex in jojoba.

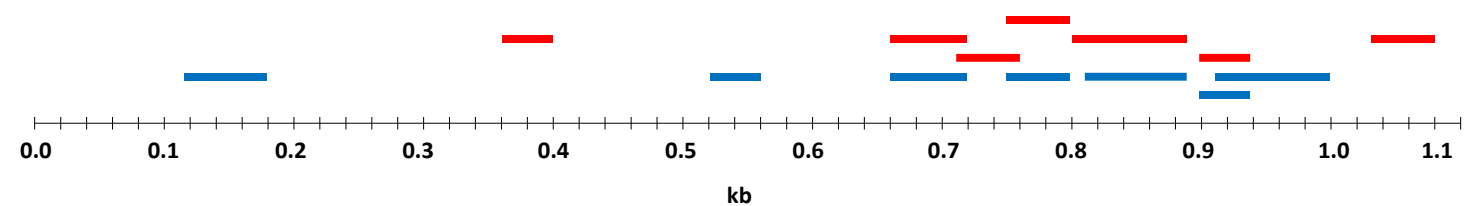

Figure 4. BLAST results for the DNA fragments within the UBC-807 1200 sequence (acc. no. HQ166029) existed in the male (blue) and female (red) de novo assembled genomes of jojoba growing in Saudi Arabia

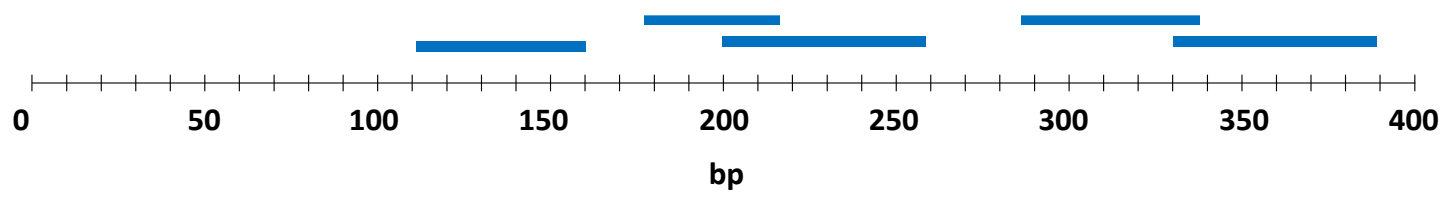

Figure 5. BLAST results for the DNA fragments within the SRAP male-specific marker (396 bp) existed in the male de novo assembled genome of jojoba germplasm growing in Saudi Arabia

\section{Conclusion}

Overall results of the present study indicated the possibility to utilize CAPS with either restriction enzymes TaqI or Bsp119I in detecting sex-specific markers in jojoba. Also, AFLP resulted in the recovery of several sex-specific markers, thus, seems to be very useful in separating the two sexes in jojoba very efficiently. The previously described male-specific SRAP marker was also confirmed in jojoba plants growing in Saudi Arabia. These three types of sex-specific markers might be useful in the future industry of biofuel production in Saudi Arabia and the Arab region. The study also refers to the necessity to barcode this important plant in different regions of the country and re-validate the recovered sex-specific markers way before we decide to incorporate this plant in breeding program or at commercial scale.

Acknowledgements. This project was funded by the Deanship of Scientific Research (DSR), King Abdulaziz University, Jeddah, under Grant no. (G: 368-247-1439). The authors, therefore, acknowledge with thanks DSR technical and financial support. 


\section{REFERENCES}

[1] Akagi, T., Henry, I. M., Tao, R., Comai, L. (2014): A Y-chromosome-encoded small RNA acts as a sex determinant in persimmons. - Science 346: 646-650.

[2] Akagi, T., Henry, I. M., Kawai, T., Comai, L., Tao, R. (2016): Epigenetic regulation of the sex determination gene MeGI in polyploid persimmon. - Plant Cell 28: 2905-2915.

[3] Akagi, T., Henry, I. M., Ohtani, H., Beppu, K., Kataoka, I., Tao, R. (2018): A Y-encoded suppressor of feminization arose via lineage-specific duplication of a cytokinin response regulator in kiwifruit. - Plant Cell 30: 780-795.

[4] Agrawal, V., Sharma, K., Gupta, S., et al. (2007): Identification of sex in Simmondsia chinensis (Jojoba) using RAPD markers. - Plant Biotechnol. Rep. 1: 207-210.

[5] Agarwal, M., Shrivastava, N., Padh, H. (2008): Advances in molecular marker techniques and their applications in plant sciences. - Plant Cell Rep. 27: 617-631.

[6] Agarwal, M., Shrivastava, N., Padh, H. (2011): Development of sex-linked AFLP markers in Simmondsia chinensis. - Plant Breed. 130: 114-116.

[7] Alstrom-Rapaport, C., Lascoux, M., Wang, Y. C., Roberts, G., Tuskan, G. A. (1998): Identification of a RAPD marker linked to sex determination in the basket willow (Salix viminalis L.). - J. Heredity 89: 44-49.

[8] Benzioni, A. (1995): Jojoba domestication and commercialization in Israel. - Horti. Rev. 17: 233-266.

[9] Benzioni, A., Mills, D., Van Boven, M., Cokelaere, M. (2005): Effect of genotype and environment on the concentration of simmondsin and its derivatives in jojoba seeds and foliage. - Industrial Crops and Products 21: 241-249.

[10] Charlesworth, D. (2016): Plant sex chromosomes. - Annu. Rev. Plant Biol. 67: 397-420.

[11] Chaves-Bedoya, G., Nunenz, V. (2007): A SCAR marker for the sex types determination in Colombian genotypes of Carica papaya. - Euphytica 153: 215-220.

[12] Coates, W., Ayerza, R., Palzkill, D. (2006): Supplemental pollination of jojoba - a means to increase yields. - Industrial Crops and Products 24: 41-45.

[13] Culley, T. M., Wolfe, A. D. (2000): Population genetic structure of the cleistogamous plant species Viola pubescens Aiton (Violaceae), as indicated by allozyme and ISSR molecular markers. - J. Heredity 86: 545-556.

[14] Danilova, T. V., Karlov, G. I. (2006): Application of inter simple sequence repeat (ISSR) polymorphism for detection of sex-specific molecular markers in hop (Humulus lupulus L.). - Euphytica 151: 15-21.

[15] Deputy, J. C., Ming, R., Ma, H., Liu, Z., Fitch, M. M., et al. (2002): Molecular markers for sex determination in papaya (Carica papaya, L.). - Theor. Appl. Genet. 106: 107-111.

[16] El-Mallah, M. H., El-Shami, S. M. (2009): Investigation of liquid wax components of Egyptian jojoba seeds. - J. Oleo Sci. 58(11): 543-548.

[17] Flachowsky, H., Schumann, E., Weber, W. E., Peil, A. (2001): Application of AFLP for the detection of sex specific markers in hemp. - Plant Breed. 120: 305-309.

[18] Gangopadhyay, G., Roy, S. K., Ghose, K., et al. (2007): Sex detection of Carica papaya and Cycas circinalis in pre-flowering stage by ISSR and RAPD. - Curr. Sci. 92: 524-526.

[19] Gawel, N. J., Jarret, R. L. (1991): A modified CTAB DNA extraction procedure for Musa and Ipomoea. - Plant Mol. Biol. Rep. 9: 262-266.

[20] Harkess, A., Zhou, J., Xu, C., et al. (2017): The asparagus genome sheds light on the origin and evolution of a young y chromosome. - Nat. Commun. 8: 1279.

[21] Hormaza, J. I., Dollo, L., Polito, V. S. (1994): Identification of a RAPD marker linked to sex determination in Pistachia vera using bulked segregant analysis. - Theor. Appl. Genet. 89: 9-13.

[22] Heikrujam, M., Sharma, K., Kumar, J., Agrawal, V. (2014): Validation of male sexspecific UBC-8071200 ISSR marker and its conversion into sequence tagged sites marker in Jojoba: a high precision oil yielding dioecious shrub. - Plant Breed. 133: 666-671. 
[23] Henry, I. M., Akagi, T., Tao, R., Comai, L. (2018): One hundred ways to invent the sexes: theoretical and observed paths to dioecy in plants. - Annu. Rev. Plant Biol. 69: 553-575.

[24] Ince, A. G., Karaca, M. (2011): Early determination of sex in jojoba plant by CAPS assay. - J Agric. Sci. 149: 327-336.

[25] Ince, G. A., Karaca, M., Onus, A. N. (2010): A reliable gender diagnostic PCR assay for jojoba (Simmondsia chinensis (Link) Schneider). - Genet. Resour. Crop Evol. 57: 773779.

[26] Jhang, T., Kaur, M., Kalia, P., Sharma, T. R. (2010): Efficiency of different marker systems for molecular characterization of subtropical carrot germplasm. - J. Agric. Sci., Cambridge 148: 171-181.

[27] Kafkas, S., Cetiner, S., Perl-Treves, R. (2001): Development of sex-associated RAPD markers in wild Pistacia species. - J. Hort. Sci. Biotechnol. 76: 242-246.

[28] Karaca, M., Ince, A. G. (2008): Minisatellites as DNA markers to classify bermudagrasses (Cynodon spp.): confirmation of minisatellite in amplified products. - J. Genet. 87: 83-86.

[29] Karaca, M., Saha, S., Callahan, F. E., et al. (2004): Molecular and cytological characterization of a cytoplasmic-specific mutant in pima cotton (Gossypium barbadense L.). - Euphytica 139: 187-197.

[30] Kazama, Y., Ishii, K., Aonuma, W., et al. (2016): A new physical mapping approach refines the sex-determining gene positions on the Silene latifolia Y-chromosome. - Sci. Rep. 6: 18917.

[31] Khadka, D. K., Nejidat, A., Tal, M., Golangoldhirsh, A. (2002): DNA markers for sex: molecular evidence for gender dimorphism in dioecious Mercurialis annua L. - Mol. Breed. 9: 251-257.

[32] Kumar, J., Heikrujam, M., Sharma, K., Agrawal, V. (2019): SRAP and SSR markerassisted genetic diversity, population structure analysis and sex identification in Jojoba (Simmondsia chinensis). - Industrial Crops and Products 133: 118-132.

[33] Kurtz, S., Phillippy, A., Delcher, A. L., et al. (2004): Versatile and open software for comparing large genomes. - Genome Biol. 5: R12.

[34] Li, M., Yang, H., Li, F., et al. (2010): A male-specific SCAR marker in Calamus simplicifolius, a dioecious rattan species endemic to China. - Mol. Breed. 25: 549-551.

[35] Liu, Z., Moore, P. H., Ma, H., et al. (2004): A primitive Y chromosome in papaya marks incipient sex chromosome evolution. - Nature 427: 348-352.

[36] Ming, R., Bendahmane, A., Renner, S. S. (2011): Sex chromosomes in land plants. Annu. Rev. Plant Biol. 62: 485-514.

[37] Mohasseb, H. A. A., Moursy, H. A., El-Bahr, M. K., et al. (2009): Sex determination of jojoba using RAPD markers and sry gene primer combined with RAPD primers. - Res. J. Cell Mol. Biol. 3: 102-112.

[38] Muyle, A., Shearn, R., Marais, G. A. (2017): The evolution of sex chromosomes and dosage compensation in plants. - Genome Biol. Evol. 9: 627-645.

[39] Mwase, W. F., Erik-Lid, S., Bjørnstad, A., Stedje, B., Kwapata, M. B., Bokosi, J. M. (2007): Application of amplified fragment length polymorphism (AFLPs) for detection of sex-specific markers in dioecious Uapaca kirkiana Muell. - Årg. Afr. J. Biotechnol. 6: 137-142.

[40] Parrish, T. L., Koelewijn, H. P., van Dijk, P. J. (2004): Identification of a male-specific AFLP marker in a functionally dioecious fig, Ficus fulva Reinw. ex Bl. (Moraceae). Sex. Plant Reprod. 17: 17-22.

[41] Passerini, E., Lombardo, P. (2000): Cosmetics. - Cosmet. News 22: 396-398.

[42] Peil, A., Flachowsky, H., Schumann, E., Weber, W. E. (2003): Sex-linked AFLP markers indicate a pseudoautosomal region in hemp (Cannabis sativa L.). - Theor. Appl. Genet. 107: 102-109. 
[43] Pinzi, S., Garcia, I. L., Lopez-Gimenez, F. J., et al. (2009): The ideal vegetable oil-based biodiesel composition: a review of social, economical and technical implications. Energy Fuels 23: 2325-2341.

[44] Prakash, S., Van Staden, J. (2006): Sex identification in Encephalartos natalensis (Dyer and Verdoorn) using RAPD markers. - Euphytica 152: 197-200.

[45] Reamson-Büttner, S. M., Schondelmaier, J., Jung, C. (1998): AFLP markers tightly linked to the sex in Asparagus officinalis. - Mol. Breed. 4: 91-98.

[46] Renner, S. S. (2014): The relative and absolute frequencies of angiosperm sexual systems: dioecy, monoecy, gynodioecy, and an updated online database. - Am. J. Bot. 101: 1588-1596.

[47] Ruas, C. F., Fairbanks, D. J., Evans, R. P., et al. (1998): Male-specific DNA in the dioecious species Atriplex garrettii (Chenopodiaceae). - Am. J. Bot. 85: 162-167.

[48] Sharma, K., Agrawal, V., Gupta, S., et al. (2008): ISSR marker-assisted selection of male and female plants in a promising dioecious crop: jojoba (Simmondsia chinensis). - Plant Biotechnol. Rep. 2: 239-243.

[49] Shibu, M. P., Ravishankar, K. V., Anand, L., et al. (2000): Identification of sex-specific DNA markers in the dioecious tree, nutmeg (Myristica fragrans Houtt.). - PGR Newslet. 121: 59-61.

[50] Tan, H., Callahan, F. E., Zhang, X. D., et al. (2003): Identification of resistance gene analogs in cotton (Gossypium hirsutum L.). - Euphytica 134: 1-7.

[51] Tobares, L., Frati, M., Guzman, C., Maestri, D. (2004): Agronomical and chemical traits as descriptors for discrimination and selection of jojoba (Simmondsia chinensis) clones. Industrial Crops and Products 19: 107-111.

[52] Torjek, O., Bucherna, N., Kiss, E., et al. (2002): Novel male-specific molecular markers (MADC5, MADC6) in hemp. - Euphytica 127: 209-218.

[53] Vaknin, Y., Mills, D., Benzioni, A. (2003): Pollen production and pollen viability in male jojoba plants. - Industrial Crops and Products 18: 117-123.

[54] Wang, J., Na, J. K., Yu, Q., et al. (2012): Sequencing papaya X and Yh chromosomes reveals molecular basis of incipient sex chromosome evolution. - Proc. Natl. Acad. Sci. USA 109: 13710-13715.

[55] Wang, Z., Jiao, Z., Xu, P., et al. (2013): Bisexual flower ontogeny after chemical induction and berry characteristics evaluation in male Vitis amurensis. - Rupr. Sci. Hortic. 162: 11-19.

[56] Xu, W. J., Wang, B. W., Cui, K. M. (2004): RAPD and SCAR markers linked to sex determination in Eucommia ulmoides Oliv. - Euphytica 136: 233-238.

[57] Yang, H. -W., Akagi, T., Kawakatsu, T., Tao, R. (2019): Gene networks orchestrated by MeGI: a single-factor mechanism underlying sex determination in persimmon. - Plant J. 98: 97-111.

\section{ELECTRONIC APPENDIX}

Appendix 1. List of RAPD (Operon Technologies, USA) and ISSR primers along with their nucleotide sequences as well as sex-specific primers generated from NUCmer

Appendix 2. Banding patterns randomly selected for RAPD (a) and ISSR (b) analyses as well as those generated from NUCmer $(c \& d)$ in a trial to detect new sex-specific markers. As models, amplicons of two RAPD (with A02 and B09 primers) and two ISSR (with 544A and HB11 primers) are shown. (c) Patterns generated from 35 reactions $(R x)$ of one male and female samples, (d) patterns of five male and five female samples for selected reactions. $M \mathrm{w}=50 \mathrm{bp}$ DNA Step ladder, $1-5=M$, 6-10 = female, $M=$ male, $F=$ female. Primer sequences are shown in Appendix 1 
Appendix 3. Forward J888 marker sequences of five male and five female samples and multiple sequence alignments using Clostal Omega

Appendix 4. Reverse J888 marker sequences of five male and five female samples and multiple sequence alignments using Clostal Omega

Appendix 5. Restriction map of J888 marker for the male sample

Appendix 6. Restriction map of $J 888$ marker for the female sample

Appendix 7. Virtual gel describing predicted restriction analyses of ClaI, HindIII, HinfI, TaqI and Bsp119I of the $888 \mathrm{bp}$ amplicon for one male and one female samples in jojoba using

SnapGene ${ }^{\circledR}$ software

Appendix 8. AFLP analysis for the primer combination EcoRI-GC/MseI-GCG in five male and five female samples in jojoba

Appendix 9. AFLP analysis for the primer combination EcoRI-TAC/MseI-GCG in five male and five female samples in jojoba

Appendix 10. BLAST analysis of the de novo assembled contigs of the jojoba male genome against the ISSR male-specific marker namely UBC-807 1200 generated by PCR with primer $U B C-807$

Appendix 11. BLAST analysis of the de novo assembled contigs of the jojoba female genome against the ISSR male-specific marker namely UBC-807 ${ }_{1200}$ generated by PCR with primer $U B C-807$

Appendix 12. BLAST analysis of the de novo assembled contigs of the jojoba male genome against the SRAP male-specific marker (396 bp) generated by PCR with primer combination Em14/Me10

Appendix 13. BLAST analysis of the de novo assembled contigs of the jojoba female genome against the SRAP male-specific marker (396 bp) generated by primer combination Em14/Me10

Appendix 14. BLAST results of the de novo assembled contigs of male jojoba plant against the published sequences of the OGI gene

Appendix 15. BLAST results of the de novo assembled contigs of male jojoba plant against the published sequences of the MeGI gene

Appendix 16. BLAST results of the de novo assembled contigs of female jojoba plant against the published sequences of the OGI gene

Appendix 17. BLAST results of the de novo assembled contigs of female jojoba plant against the published sequences of the MeGI gene 